\title{
DOSSIER
}

SANDRINE BERGER-DOUCE

IAE de Valenciennes

\section{Le développement durable, un levier dPinnovation pour les PME ?}

Le développement durable (DD) constitue un levier d'innovation pour les organisations indépendamment de leur taille. Basé sur l'étude de cas exploratoire d'une PME industrielle du Nord de la France, I'article ${ }^{1}$ montre comment le $D D$ peut être un vecteur de la capacité dynamique d'innovation en milieu PME. Ce type de capacité dynamique renvoie à la conciliation d'innovations d'exploration et d'exploitation. 
$\mathrm{L}$ es enjeux environnementaux (changement climatique, raréfaction des ressources naturelles) associés à des pressions sociétales de plus en plus vives constituent un milieu favorable à l'exploitation d'opportunités d'innovation (Abrassart et Aggeri, 2007). Parallèlement, les stratégies d'innovation sont reconnues comme gages de la pérennité des organisations, indépendamment de leur taille (CamisonZomosa et al., 2004 ; Soparnot et Stevens, 2007). Au-delà d'être un levier pour gagner en acceptabilité et en légitimité, les stratégies de développement durable (DD), définies comme des stratégies visant une performance globale (Martinet et Payaud, 2008) incluant les aspects sociaux et environnementaux, représentent de véritables opportunités économiques pour les entreprises. Pour Mc Williams et al. (2006), la RSE comme traduction managériale du DD est une «forme d'investissement stratégique ». Par ailleurs, un intérêt croissant se manifeste pour les recherches sur les organisations ambidextres capables de concilier innovations d'exploitation et d'exploration dans une optique de survie (Chanal et Mothe, 2005). Si les travaux portant sur les grandes entreprises sont nombreux, le contexte des PME demeure encore largement ignoré par les recherches académiques en dépit de l'importance économique et du dynamisme de ces entreprises (Morsing et Perrini, 2009).

L'objet de cet article est de mieux comprendre comment le DD peut être un vecteur de la capacité dynamique (Teece et al., 1997) d'innovation définie comme la conciliation d'innovations d'exploitation et d'exploration dans le contexte spécifique des PME. Pour ce faire, une étude de cas unique (Yin, 1994) est privilégiée afin de saisir en profondeur les réalités multiples d'une attitude résolument proactive d'un entrepreneur militant. La première partie de l'article précise la notion de capacité dynamique d'innovation inspirée des principes du DD dans un contexte de PME. La seconde partie analyse le cas d'une PME du Nord de la France illustrant la manière dont un management responsable peut s'inscrire dans une démarche d'innovation stratégique.

\section{I - DÉVELOPPEMENT DURABLE ET STRATÉGIES D'INNOVATION EN MILIEU PME}

\section{Les PME oubliées des stratégies de DD ?}

\section{Des stratégies de DD au service d'une valeur partagée}

La littérature recense plusieurs typologies en matière de stratégie de DD. Bellini (2003) oppose la logique additive (où l'entreprise ne remet pas en cause son processus de décision) à la logique systémique (où l'intégration de l'environnement modifie la structure de décision en profondeur). Sur cette base, l'auteur distingue les comportements écodéfensifs (suivant une logique exclusivement financière), les comportements écoconformistes (se contentant de respecter les normes imposées par la réglementation) et les comportements écosensibles (ou proactifs) (allant au-delà des règlementations en vigueur). Bellini (2003) précise que seuls les comportements écosensibles relèvent de la stratégie d'entreprise. Martinet et Reynaud (2004) identifient trois types d'attitudes stratégiques :

1) une attitude attentiste relative à l'absence de volonté de s'impliquer de la part du dirigeant ou, de manière plus pragmatique, à l'insuffisance des ressources disponibles ; 
2) une attitude adaptative visant à se conformer à la législation ou à devancer les attentes futures du marché par une offre « responsable » et

3) une attitude proactive ayant pour ambition de jouer un rôle de pionnier (d'innovateur) au sein d'un secteur d'activité.

En croisant ces stratégies de DD avec les technologies environnementales déployées dans les organisations, Mathieu (2008) distingue une approche adaptative caractérisée par des innovations environnementales incrémentales d'une approche proactive, seule digne de faire progresser l'entreprise sur la voie du DD (Hart, 1995). Les stratégies proactives demeurent largement minoritaires car elles supposent que les organisations disposent des outils de gestion adaptés et de moyens financiers importants (Mathieu et Soparnot, 2007). Les entreprises adoptant une stratégie proactive visent une performance à long terme basée sur des réductions de coûts et une quête de légitimité. Ces stratégies renvoient à la RSE stratégique de Porter et Kramer (2006) : « Pour ces entreprises, la donnée écologique/sociale est stratégique. L'écologie et le social ne sont plus considérés comme des critères décisionnels, mais comme des finalités de l'action au même titre que l'économique » (Mathieu et Soparnot, 2007), mais également à la RSE intégrée (Martinet et Payaud, 2008) qui « concerne des actions en relation avec les activités de l'entreprise, c'est-à-dire proches du cœur de métier » (p. 203). Ces démarches proactives sont susceptibles de contribuer à la construction d'un potentiel de création de valeur partagée au sens de Porter et Kramer (2006, 2011). La notion de valeur partagée se définit comme « l'ensemble des politiques et des pratiques assurant la compétitivité d'une entreprise tout en développant les conditions économiques et sociales des communautés auprès desquelles elle opère » (2011, p. 66). Selon ces auteurs, créer de la valeur partagée peut emprunter trois voies : 1) reconcevoir les produits et les marchés ; 2) redéfinir la productivité au sein de la chaîne de valeur et 3 ) permettre le développement de « clusters » locaux. Or, Porter et Kramer (2011) reconnaissent que beaucoup de pionniers de la mise en pratique de cette valeur partagée sont des entrepreneurs disposant de ressources limitées, notamment dans le champ de l'entrepreneuriat social. Dans cette optique, il semble légitime de postuler que les PME sont dotées de caractéristiques favorables à cette posture de création de valeur partagée.

\section{Quelles réalités pour ces stratégies de DD en milieu PME ?}

Les stratégies de DD les plus connues émanent surtout des grandes entreprises (Conceiçao et al., 2006), oubliant presque la multitude d'actions menées en la matière dans les PME (Morsing et Perrini, 2009), d'où les appels à intensifier les recherches académiques (Moore et Spence, 2006). Sur la base de ses travaux antérieurs, Spence (2007) dresse l'inventaire des caractéristiques des stratégies de DD dans les PME. Outre un manque de formalisation, cet auteur relève l'importance de la motivation personnelle du dirigeant d'autant plus forte s'agissant d'un propriétairedirigeant, la personnalisation des relations associée à un ancrage local créant une «proximité morale » avec les communautés environnantes, la place privilégiée accordée aux salariés comme parties prenantes principales (Hammann et al., 2009), sans oublier l'impact du secteur d'activité 
sur l'engagement responsable. Au-delà des constats, des auteurs comme Jenkins (2006) dans son étude de vingt-quatre PME " championnes de RSE » ont suggéré des démarches pour inciter les PME à davantage s'engager dans cette voie.

Souvent, les initiatives des PME souffrent d'un manque de légitimité en raison de leur moindre visibilité (Jenkins, 2004). Ces entreprises ne sont pourtant pas absentes de ce mouvement (Observatoire des PME européennes, 2002), mais restent souvent timides lorsqu'il s'agit de communiquer sur le sujet (Murillo et Lozano, 2006), particulièrement vis-à-vis de leurs parties prenantes externes (Nielsen et Thomsen, 2009). Selon ces auteurs, le management responsable renvoie à un ensemble de pratiques influencées principalement par les valeurs et les convictions personnelles des dirigeants, rejoignant ainsi Murillo et Lozano (2006), Spence (2007) et Jenkins (2009). Déjà en 1999, MacLagan envisageait le management responsable comme un processus permettant l'articulation des valeurs éthiques des propriétaires dirigeants de PME avec leurs préoccupations managériales.

\section{L'articulation entre DD et innovation, une opportunité pour les PME}

\section{DD, innovation et ambidextrie}

L'innovation constitue l'un des facteurs essentiels de la performance des organisations (Soparnot et Stevens, 2007). Sur le terrain, l'enquête communautaire sur l'innovation (ECI) utilise la définition de l'innovation issue du Manuel d'Oslo ${ }^{2}$ (version 2005). Quatre catégories d'innovations sont ainsi identifiées : 1) de produit ou de prestation (entreprise du commerce ou des services) ; 2) de procédé (mise en œuvre de nouvelles techniques pour la production de biens ou la réalisation de prestations de services); 3) d'organisation (exemple des cercles de qualité) et 4) de marketing (exemple de la communication sur internet). Cette approche se retrouve dans la littérature académique qui répertorie les innovations selon leur nature (innovation de produit, innovation de procédé, innovation commerciale et innovation organisationnelle) (Barreyre, 1975) et leur degré de radicalité (innovation de rupture et innovation d'adaptation de l'existant) (Damanpour, 1991).

Au-delà des formes de l'innovation, Schumpeter insistait dès 1935 sur l'importance de l'innovation technologique. La montée en puissance du DD dans les analyses sociopolitiques contribue à la multiplication des réflexions sur le lien entre innovation (notamment technologique) et DD (Patris et al., 2001, Mathieu, 2008, Castiaux, 2009). À titre d'exemple, Rennings et al. (2006) ont montré l'existence d'une relation positive entre le degré de maturité du système de management environnemental déployé au sein d'une entreprise et ses processus d'innovation technologique. Autrement dit, le DD n'est plus uniquement appréhendé comme un possible réducteur de coûts pour les organisations, mais davantage comme un catalyseur d'innovation (Grayson et Hodges, 2004 ; Little, 2006 ; Nidumolu et al., 2009) porteur de création de valeur partagée (Porter et Kramer, 2011). Selon l'étude de March (1991) sur l'apprentissage organisationnel, la survie et la prospérité d'une organisation reposent sur

2. Le Manuel d'Oslo est présenté comme la principale source internationale de principes directeurs en matière de collecte et d'utilisation d'informations sur les activités d'innovation (source : site internet de l'Insee). 
un savant équilibre entre l'exploitation d'activités déjà maîtrisées et l'exploration de nouvelles voies ${ }^{3}$. S'appuyant sur le courant des capacités dynamiques en management stratégique (Teece et al., 1997 ; Eisenhardt et Martin, 2000), Chanal et Mothe (2005) définissent une capacité dynamique d'innovation comme la capacité à combiner innovations d'exploitation et d'exploration. Ainsi, l'organisation peut mener de front des projets de « conception de nouveaux produits ou services en s'appuyant essentiellement sur les compétences existantes de l'entreprise » (innovation d'exploitation) et des projets «d'exploration qui se déploient davantage dans une logique de rupture et de création de nouvelles connaissances et compétences stratégiques »(p. 174). Ces auteurs ouvrent ainsi la voie à des recherches sur l'organisation ambidextre, une notion empruntée à Duncan (1976) et diffusée par Gupta et al. (2006), qui semble s'imposer aux organisations, y compris aux PME. L'identification de cette tension entre exploitation et exploration déjà présente chez Schumpeter (1935) dans sa distinction entre les « exploitants purs et simples » et les entrepreneurs, est reprise dans la typologie de comportements liant responsabilité et innovation de Castiaux (2009).

\section{Spécificités des PME au regard de l'innovation}

L'innovation constitue l'un des facteurs clés de développement stratégique des PME (Aragon-Sanchez et Sanchez-Marin, 2005). Pour Julien et Lachance (2006), « L'entrepreneuriat, c'est d'abord une création de valeurs collectives, reconnues par les divers acteurs qui partagent les défis et les risques de la novation acceptée par le marché proche, par le milieu et finalement par l'extérieur ». Dans cette optique, l'intégration du DD dans le domaine de l'entrepreneuriat peut s'envisager comme la construction et l'exploitation d'une opportunité sous la forme d'une innovation, de la création d'une nouvelle organisation ou de valeur.

Selon Ayerbe (2006), les caractéristiques organisationnelles des PME ont tendance à favoriser l'innovation. Aussi, les études sur l'influence des profils d'entrepreneurs sur leur management ont fait l'objet de nombreuses publications. S'agissant de l'attitude face à l'innovation, Miles et Snow (1978) distinguent quatre types d'entrepreneurs : 1) le prospecteur, un innovateur pur (la création est son principal centre d'intérêt, alors que le suivi et le développement l'intéressent beaucoup moins) ; 2) l'innovateur, également créateur, avec une volonté d'engager un processus plus complet de développement ; 3) le suiveur qui n'innove pas, mais se contente d'imiter et 4) le réacteur qui s'adapte toujours après coup et de manière très ponctuelle. Déjà en 1975, les travaux de Barreyre soulignaient une spécificité des stratégies d'innovation dans les $\mathrm{PME}$, à savoir une logique d'action fondée sur les intentions propres des entrepreneurs. Par analogie, nous postulons que les PME présentent des spécificités intrinsèques susceptibles de favoriser l'ambidextrie comme un fonctionnement plutôt intuitif, un comportement propice à la prise de risques, une flexibilité par rapport aux changements de

3. Sur les notions d'innovations d'exploration et d'exploitation, voir le dossier coordonné par C. Mothe et S. Brion dans la Revue française de gestion (vol. 34, $\mathrm{n}^{\circ} 187,2008$ ). 
l'environnement, une indépendance financière grâce à la présence du propriétairedirigeant ou encore la spécialisation des activités (Julien, 1997). Pour Jenkins (2009), ces caractéristiques peuvent aider les PME à saisir des opportunités en termes de DD. Selon elle, l'innovation est un élément clé de toute PME impliquée dans une RSE stratégique (Porter et Kramer, 2006).

\section{Un questionnement sur les relations entre innovation et DD en milieu PME}

Les relations entre innovation et DD sont peu étudiées dans la littérature académique (Castiaux, 2009), alors même que Porter, dès 1991, affirmait que la RSE pouvait stimuler l'innovation. La prise de conscience environnementale est ainsi présentée par Porter et Van der Linde (1995) comme potentiellement initiatrice d'innovations créatrices de valeur. Plus récemment, Grayson et Hodges (2004), Rennings et al. (2006) ou encore Little (2006) ont abouti à des résultats comparables. En 2010, Le Bas et Poussing ont montré que les entreprises luxembourgeoises technologiquement innovantes adoptaient, davantage que les autres, un management responsable. Ces travaux soulignent toute la complexité des relations entre innovation et DD placées sous le signe d'une réelle interdépendance.

En dépit de son importance, cette question ne se retrouve posée que dans quelques études européennes portant sur le terrain des PME. Kramer et al. (2007) ont étudié une cinquantaine de PME danoises engagées dans des pratiques responsables. Ces auteurs concluent sur le lien entre innovations « durables » (tant sociales qu'environnementales) et performance économique

\section{MÉTHODOLOGIE}

La méthodologie de recherche est de nature qualitative (Hlady Rispal, 2002) en raison du caractère émergent de la problématique à traiter (Wacheux, 1986). Une recherche qualitative est par essence un travail d'artisanat (Wacheux, 1996) : «La mise en œuvre d'un processus de recherche qualitatif, c'est avant tout vouloir comprendre le pourquoi et le comment des événements dans des situations concrètes. » S'agissant d'un thème émergent, nous avons privilégié une méthodologie qualitative centrée sur une étude de cas unique (Yin, 1994 ; HladyRispal, 2002). L'unité d'analyse, au sens de Yin (1994), est ici la PME. L'étude de cas a été constituée sur la base de traitement de données secondaires : articles de presse, sites internet, documents internes (discours des conventions de 2007 et 2009), témoignages lors d'événements professionnels (Journée annuelle DD et entreprises 2008 à Lille et tour de France de l'ISO 26000 en mai 2010 à Arras) et de données primaires. Ces dernières ont été collectées lors de trois entretiens semi-directifs avec le dirigeant (durée moyenne de deux heures) portant sur : 1) les activités de l'entreprise, 2) les pratiques en faveur du DD, 3) le parcours et les convictions du dirigeant, 4) la vision stratégique du dirigeant (repreneur) et d'entretiens non directifs avec des salariés (responsable des ventes, responsable du marketing, responsable qualité-sécurité-environnement, chef d'équipe, assistante qualité, deux stagiaires) (durée moyenne d'une heure), ainsi que lors d'une visite du site industriel durant l'été 2010. 
mesurée par le gain en parts de marché de ces PME. L'étude de Mendibil et al. (2007) (citée par Castiaux, 2009) sur des PME innovantes en Espagne, en Italie et au Royaume-Uni montre une réelle proximité entre innovation et DD, même si les causalités respectives demeurent difficiles à évaluer. Cependant, un fort engagement vis-àvis des salariés et des parties prenantes externes apparaît comme un gage de compétitivité à l'aune des capacités d'innovation de ces entreprises. Jenkins (2009) propose un modèle d'opportunités d'affaires de la RSE en milieu PME en cinq étapes mobilisant la notion de capacités dynamiques (Teece et al., 1997) et qui accorde une place privilégiée à l'innovation.

Au regard de l'état de l'art, nous proposons la problématique de recherche suivante : comment le développement durable peut-il être un vecteur de la capacité dynamique d'innovation définie comme la conciliation d'innovations d'exploitation et d'exploration dans les PME?

\section{II - UNE ÉTUDE EMPIRIQUE DE LA CAPACITÉ DYNAMIQUE D'INNOVATION DURABLE DANS UNE PME}

\section{Présentation de la PME et de son engagement en faveur du DD}

\section{Une entreprise dynamique sur un marché en profonde mutation}

Fondée en 1928 près de Lille, la PME de 101 salariés est un fabricant d'enveloppes de mise sous pli automatique. Elle produit deux milliards d'enveloppes chaque année (soit $10 \%$ de la production nationale) pour un chiffre d'affaires de 21 millions d'euros en 2010 (22 prévus pour 2011, sur un marché pourtant en régression). Ses clients, principalement des grandes entreprises (Gaz de France, SFR, Orange, La Poste, etc.), utilisent ces enveloppes pour l'acheminement des factures et autres relevés (cas de l'assurance maladie). En 1997, après neuf années d'expérience professionnelle dans de grandes entreprises, le dirigeant décide de changer radicalement de vie et de devenir entrepreneur à 32 ans. En 2008, il rachète l'entreprise dont il est désormais l'unique actionnaire.

Le marché de l'enveloppe est confronté à de sérieuses difficultés économiques depuis quelques années puisqu'il a régressé de $7 \%$ en volume entre 2006 et 2009, tout en continuant à progresser en valeur $(+6 \%$ entre 2007 et 2008), essentiellement grâce au développement des produits à forte valeur ajoutée ${ }^{4}$. «Le marché n'est pas stable et il faut développer des avantages concurrentiels » (responsable QSE). L'appel croissant à la dématérialisation des factures et des prospections dans les entreprises n'est pas étranger à cette évolution. C'est dans ce contexte que la PME a commandé en 2009 à un cabinet indépendant une analyse du cycle de vie ${ }^{5}$ de l'enveloppe dont les résultats validés par le CNRS et publiés en mai $2010^{6}$ sont édifiants. L'envoi d'une facture de deux pages par voie postale produit $15,7 \mathrm{~g}$ de $\mathrm{CO}_{2}$ tandis que son avatar virtuel

4. Source : « Le papetier de France », juin-juillet 2009, p. 54-59.

5. Cette ACV a mesuré les impacts des deux modes d'envoi selon sept indicateurs : le bilan carbone, l'épuisement des ressources non renouvelables, l'acidification de l'air, l'eutrophisation de l'eau, la destruction de la couche d'ozone, la toxicité humaine et la consommation d'énergie cumulée.

6. Une synthèse des résultats est disponible sur le site internet de la PME. 
(le courrier électronique) en produit 15 fois plus, soit $252 \mathrm{~g}$. En résumé, agir en faveur de l'environnement incite à promouvoir la filière papier, contrairement à une idée reçue. Ainsi, une véritable offensive écologique s'organise depuis quelques années sur le marché de l'enveloppe. Ces produits à forte valeur ajoutée sont le fruit des stratégies d'innovation des fabricants impulsées par la responsabilité de la profession s'agissant des enjeux écologiques. La matière première de ces industriels (la pâte à papier) contribue à renouveler la forêt qui augmente actuellement de 50000 hectares par an en France ${ }^{7}$. C'est dans ce contexte que le dirigeant de la PME a décidé de revoir son modèle économique. « Le problème, c'est que l'enveloppe est un produit parfaitement banal qui n'a pas de prix ou plutôt uniquement un prix qui baisse. Voilà en résumé l'idée de mon prédécesseur. Or, l'entreprise disposait d'une force extraordinaire, le savoir-faire de l'équipe. Donc, l'objectif était de sortir de la banalité du produit. Et là, l'idée de mettre du DD dans la réflexion prenait tout son sens... Enfin, je pouvais être en phase avec mes convictions profondes. " (dirigeant). Ainsi, concrètement, à chaque arbre coupé, trois sont replantés.

\section{La stratégie DD de la PME}

La stratégie DD de la PME se décline en principes dont les cinq rubriques sont :

1) Pérennité, durabilité, finances saines: réinvestissement intégral $\mathrm{du}$ résultat net dans l'entreprise selon la répartition suivante : un tiers en prime exceptionnelle, un tiers dédié à la rénovation du site industriel et un tiers consacré à la maintenance et à la rénovation du matériel. Environ $10 \%$ du chiffre d'affaires est investi en matériels et en recherche $\&$ développement, ce qui permet à la PME de disposer des technologies les plus performantes dans sa spécialité. « Outre une qualité régulière de nos produits et services, nos clients attendent de nous la sécurité produite par des finances saines qui engagent la pérennité de l'offre et de l'entreprise ${ }^{8}$.

2) Hygiène, sécurité et méthode de réduction des risques : les métiers de production sont dangereux et pénibles. À titre d'exemple, le papier défile dans les machines à la vitesse de 400 mètres par minute, d'où une vigilance de chaque instant. Un principe de prévention active a été retenu, en association avec un souci de réduction de la pénibilité et de la dangerosité des tâches. Cela figure explicitement dans la politique QSE 2010-2011 de la PME. Dans cette optique de réduction des risques, le site est propre et bien rangé, une manière d'assurer une circulation fluide.

3) Formation : la spécificité des métiers dans les ateliers implique une formation interne assurée par les techniciens les plus anciens de l'entreprise. Cette transmission de la connaissance technique contribue à la valorisation du savoir-faire des salariés de la PME. Dans son recrutement, le dirigeant favorise la diversité des parcours qu'il considère comme une source d'enrichissement culturel pour l'organisation (Jenkins, 2006). Des étudiants de tous horizons sont régulièrement intégrés en stage ou en contrat d'alternance. La politique de formation repose sur le développement des com-

7. Source : Les échos judiciaires Girondins, $\mathrm{n}^{\circ}$ 5556, 7 avril 2009.

8. Source : site internet de l'entreprise. 
pétences et de la polyvalence, à laquelle s'ajoute « une politique salariale diversifiée qui nous vaut d'être un vecteur d'intégration professionnelle et sociale dans la région » (responsable QSE).

4) Choix des matières premières : le papier utilisé pour la production d'enveloppes provient de forêts gérées durablement, c'est-àdire dans le respect de la biodiversité des espèces. Par sa consommation, la PME participe annuellement à la plantation ${ }^{9}$ de 180000 arbres. Les encres et les colles sont formulées à base d'eau et sans solvant depuis 1999. Depuis trois ans, la PME s'est engagée à supprimer totalement les déchets industriels banals : «On trie et on valorise tous nos déchets (encres, colles, chiffons, papiers). Par exemple, chez nous, il n'y a pas d'eau de process renvoyée dans le réseau. $50 \%$ de l'eau utilisée par l'entreprise provient de la récupération des eaux de pluie (environ $400 \mathrm{~m}^{3}$ ) et sert à l'arrosage du jardin et au fonctionnement des sanitaires. L'eau « usée » est pompée, stockée, puis envoyée dans une cimenterie à $50 \mathrm{~km}$ de l'entreprise. » (dirigeant).

5) QSE : certifications pour la qualité, la sécurité et l'environnement. La PME est certifiée ISO 9001 (qualité, depuis 1996), OHSAS 18001 (sécurité) et ISO 14001 (environnement) depuis février 2008. En 1998, l'entreprise avait participé à la création de la norme NF Environnement pour l'enveloppe en coopération avec l'Afnor. En décembre 2008, l'entreprise a obtenu les certifications $\mathrm{FSC}^{10}$ et PEFC. La politique
QSE de l'entreprise a été formalisée en avril 2009 par le dirigeant de manière à investir pour toujours améliorer la qualité des produits. Concrètement, cette politique QSE, dont la deuxième version (2010-2011) a été rédigée en juillet 2010, se traduit dans toutes les décisions d'investissement.

La stratégie DD de la PME est une illustration d'une stratégie écosensible (Bellini, 2003) caractéristique d'une attitude proactive (Martinet et Reynaud, 2004) et d'une RSE intégrée (Martinet et Payaud, 2008), proche des résultats de Jenkins (2006) sur les PME « championnes de RSE ». Le dirigeant de la PME s'attache cependant fortement à mesurer les bénéfices induits par son engagement, de manière à prouver la valeur créée. À l'avenir, il a pour ambition de développer une activité industrielle à énergie passive, autrement dit à récupérer systématiquement les énergies du process de production pour les réinjecter dans les postes consommateurs ${ }^{11}$. Ce management responsable repose sur des innovations multiples.

\section{Une stratégie d'innovation multiple}

Les innovations de la PME sont présentées selon la typologie du Manuel d'Oslo (2005).

\section{Innovations de produits}

L'innovation est omniprésente dans les produits proposés par la PME car développer une offre environnementale par l'écoconception est un moyen de créer de la valeur sur un marché en mutation : " On a à cœur de proposer à nos clients des produits tou-

9. Le nouveau site internet de la PME (mis en service en novembre 2010) propose sur sa page d'accueil un compteur en temps réel du nombre d'arbres plantés grâce aux commandes des clients de l'entreprise.

10. FSC (Forest Stewardship Council) et PEFC (Programme for the Endorsement of Forest Certification) sont des certifications visant à promouvoir une gestion durable des forêts.

11. Source : politique QSE 2010-2011 de l'entreprise. 
jours novateurs. » (responsable marketing), d'où une création de valeur partagée au profit des clients (Porter et Kramer, 2011). Les enveloppes écocompatibles représentent un marché potentiel important. En 2009, les enveloppes estampillées NF Environnement représentent environ $20 \%$ des ventes en France et parmi les enveloppes à fenêtre, seules $30 \%$ sont entièrement recyclables et biodégradables ${ }^{12}$. Depuis une dizaine d'années, la PME développe des enveloppes innovantes entièrement recyclables, produites avec des encres à base d'eau, disposant de fenêtres en papier cristal, composées de fibres naturelles non traitées et non blanchies. Toujours en quête d'innovation comme source d'avantage compétitif (Jenkins, 2009), la PME propose également une enveloppe dite " transpromotionnelle », un concept importé des pays anglo-saxons. Le document transactionnel (relevé de compte, facture) contient un message promotionnel destiné aux clients, ce qui permet de limiter les envois et donc les frais induits, une solution appréciée des entreprises en ces temps de restrictions budgétaires. Parallèlement, la PME a lancé en 2009 une marque de pochettes d'emballage personnalisables à décliner en petites séries entièrement façonnées à la main. Ces produits peuvent, par exemple, servir à des éditions limitées de pochettes de CD pour des labels indépendants. Cette offre "artisanale » combine plusieurs volets du DD, car outre le fait que les matériaux utilisés pour la fabrication des pochettes soient tous durables, le façonnage à la main est assuré par des prisonniers dans le cadre de leur projet de réin- sertion professionnelle. Cette activité sert de support à la création de valeur partagée au profit de communautés extérieures à l'organisation (Porter et Kramer, 2011), ici des prisonniers en réinsertion.

La PME ne se contente pas d'innover dans son métier de la fabrication d'enveloppes puisqu'elle s'est lancée début 2010 dans une innovation de service, en l'occurrence une diversification vers le conseil en DD aux PME, un levier de création de valeur partagée avec d'autres PME régionales (Porter et Kramer, 2011), mais aussi une manière de pérenniser l'entreprise confrontée aux turbulences du marché de l'enveloppe. Forte de son expérience du DD, la PME propose des services structurés autour du tryptique produit/process/bâtiment : analyse du cycle de vie, écoconception, préparation de l'audit QSE ou encore diagnostic énergétique : "L'idée est d'accompagner des responsables de PME dans leur réflexion en leur montrant que c'est possible d'être profitable en respectant l'environnement et en mettant l'humain au centre de ses préoccupations. » (responsable QSE).

\section{Innovations de procédés}

Dans un souci de réduction des déchets d'emballage, la PME a développé en 1999 un système de conditionnement des enveloppes en bobines réutilisables et consignées, un exemple de technologie environnementale intégrée (Patris et al., 2001). Cette technologie d'enroulage brevetée ${ }^{13}$ permet de réutiliser les jantes et chariots assurant le transport et le stockage des enveloppes. Ce procédé innovant a permis d'économiser deux millions de boîtes en

12. Source : Les échos judiciaires Girondins, $\mathrm{n}^{\circ} 5556,7$ avril 2009.

13. Le brevet est la propriété du dirigeant de la PME et de deux de ses collaborateurs dans le domaine de la production. 
cartons et 30000 palettes en dix ans. Début 2011, environ un tiers des enveloppes produites par la PME pour cinq gros clients utilisent ce système. Au-delà des économies de carburant réalisées grâce à la rationalisation des flux logistiques (source de création de valeur partagée, Porter et Kramer, 2011), cette technologie contribue à diminuer la manutention (suppression d'un poste pénible sur la chaîne) et donc les maladies professionnelles (au niveau du canal carpien des opérateurs). Par ailleurs, elle s'inscrit dans une politique plus globale de modernisation de l'outil de production: « L'automatisation est un sujet qui fait peur au personnel, car ils redoutent de perdre leur emploi. Par exemple, on avait avant des receveuses en bout de chaîne qui avaient un travail très pénible, avec au final de nombreux troubles musculo-squelettiques (TMS). Dans le cadre de la modernisation des ateliers, on a investi depuis 2003 dans des machines de mise en boîte automatique des enveloppes. Ces femmes (sans qualification) ont été formées au métier de contrôleuses qualité. » (dirigeant). Cette décision a permis la requalification de ces salariées tout en assurant un haut niveau de qualité de la fabrication et donc la performance de la PME.

\section{Innovations d'organisation}

Parallèlement, les innovations sociétales se multiplient comme la création d'une association loi 1901 : «On a aussi fondé une association qui a pour ambition de contribuer au reboisement du Nord Pas-de-Calais (la région ne compte que $7 \%$ de surfaces boisées contre $27 \%$ au niveau national). On essaye de microagir pour faire bouger les choses...» (dirigeant). L'association fédère, sensibilise et implique les habitants de la région autour de projets de reboisement de leur environnement de proximité, synonymes de création de valeur partagée (Porter et Kramer, 2011). En six mois, une dizaine de projets de plantation ont été réalisés et ont bénéficié d'une couverture médiatique régionale et nationale ${ }^{14}$.

Dans la même optique, la rénovation du site industriel s'inscrit dans une volonté d'une meilleure intégration dans l'environnement naturel de la PME tout en réalisant des économies substantielles sur les factures énergétiques. Une meilleure isolation des ateliers permet de réduire les nuisances sonores pour les riverains de l'usine tout en préservant la biodiversité locale : «On a le projet de développer une toiture végétalisée de $1100 \mathrm{~m}^{2}$ au dessus des ateliers, on a déjà installé ce type de toiture sur $220 \mathrm{~m}^{2}$ au dessus des bureaux en 2009. Au final, on espère réduire d'un tiers la facture d'électricité de l'entreprise (passer de $300 \mathrm{~K} €$ à environ $200 \mathrm{~K} €$ ). » (dirigeant). Ces bénéfices pour les riverains vont de pair avec les avantages pour les ouvriers en termes d'amélioration de leurs conditions de travail, gage d'une création de valeur partagée au profit des salariés (Porter et Kramer, 2011), tout en permettant des économies d'énergie importantes, signe d'une possible conciliation entre intérêts économiques, sociaux et environnementaux ${ }^{15}$. La rénovation du bâtiment

14. Exemples : journal télévisé du $13 \mathrm{~h}$ de TF1, article de presse économique sur le mécénat d'entreprise, reportage dans une émission sur France 3.

15. L'installation de puits de lumière au-dessus des ateliers (coût : $300 \mathrm{k} €$ ) permet de réduire de $50 \%$ la facture électrique d'éclairage et améliore le confort de travail des ouvriers. 
administratif (datant de 1848) avec la création d'un open space lumineux ouvert sur la nature améliore les conditions de travail, d'où un moindre stress, mais aussi des factures d'électricité en baisse.

\section{Innovations de marketing}

Depuis 2007, la PME développe une réelle stratégie de marque visant à améliorer la gestion de son portefeuille de marques, à l'instar des pratiques des grandes entreprises. Ce management des marques consiste à revisiter les marques détenues par l'entreprise dans une optique de plus grande cohérence et de visibilité accrue pour les clients et les salariés (machines dédiées à la fabrication de tels produits repeintes selon le code couleur des produits) (responsable marketing), avec un objectif de création de valeur partagée interne et externe (Porter et Kramer, 2011). La démarche s'accompagne de la refonte du site internet décidée au printemps 2010 dont l'un des objectifs est explicitement de clarifier l'offre de produits. Le nouveau site internet mis en ligne fin 2010 se distingue par sa convivialité et son esthétique directement inspirée de la nature avec le symbole de l'arbre comme fil directeur des différentes rubriques. La boutique en ligne est davantage mise en valeur, de même que les résultats en temps réel de l'engagement de la PME avec, sur la page d'accueil, un compteur des arbres replantés par l'entreprise grâce aux commandes de ses clients. Ces innovations constituent pour la PME une véritable révolution dans la manière d'appréhender ses marchés, signe d'une reconception profonde de son métier (Porter et Kramer, 2011).

\section{Discussion des résultats en termes d'ambidextrie et de posture entrepreneuriale}

\section{Une analyse par la capacité dynamique d'innovation durable}

L'analyse du cas de la PME montre le passage d'une gestion des risques à un management responsable par la conciliation d'innovations d'exploitation et d'exploration (figure 1). Indépendamment de leur nature, toutes les innovations déployées par le dirigeant et son équipe reposent sur des convictions ancrées dans le DD (Jenkins, 2006) mises au service de la création de valeur partagée en interne et en externe (Porter et Kramer, 2011). Ces innovations ont tendance à se renforcer mutuellement au fil des années, donnant potentiellement naissance à un cercle vertueux. La démarche industrielle de l'entreprise repose sur l'anticipation permanente des besoins portée par la triple certification QSE de la PME dans un souci d'amélioration continue. Par exemple, la technologie d'enroulage brevetée par la PME, outre des économies par la suppression de deux millions de boîtes en cartons en dix ans, contribue à la diminution de la pénibilité des tâches pour les opérateurs de l'atelier et à la réduction des risques de maladies professionnelles. Ces opérateurs ne portent désormais plus de boîtes et ne s'occupent plus des enveloppes durant près de trois heures. La santé et la sécurité au travail renvoient à l'un des impératifs de gestion les plus incontournables et à l'un des éléments constitutifs du $\mathrm{DD}$, « dont l'intégration dans les entreprises précède celle du DD de plusieurs décennies $^{16} \gg$. Leur intégration dans le processus 
Tableau 1 - Les trois volets du DD en filigrane des innovations de la PME

\begin{tabular}{|c|c|c|}
\hline $\begin{array}{r}\text { Volets } \\
\text { du DD }\end{array}$ & Exemples & Innovations \\
\hline \multirow{4}{*}{ 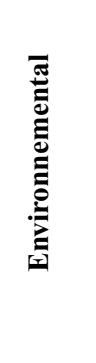 } & $\begin{array}{l}\text { Limitation des impacts } \\
\text { environnementaux à la source }\end{array}$ & $\begin{array}{l}\text { Technologie d'enroulage brevetée, récupération par aspiration } \\
\text { des déchets papier pour le centre de tri du site }\end{array}$ \\
\hline & $\begin{array}{l}\text { Utilisation de matériaux } \\
\text { naturels et labellisés }\end{array}$ & $\begin{array}{l}\text { Enveloppes éco-compatibles, enveloppes } \\
\text { transpromotionnelles, pochettes personnalisables }\end{array}$ \\
\hline & $\begin{array}{l}\text { Protection de la biodiversité } \\
\text { locale }\end{array}$ & $\begin{array}{l}\text { Rénovation des toitures par le choix de la végétalisation, } \\
\text { association pour le reboisement régional }\end{array}$ \\
\hline & $\begin{array}{l}\text { Limitation des transports des } \\
\text { eaux usées }\end{array}$ & $\begin{array}{l}\text { Bambouseraie de phytoremédiation (infiltration des eaux } \\
\text { usées sur place) }\end{array}$ \\
\hline \multirow{6}{*}{ 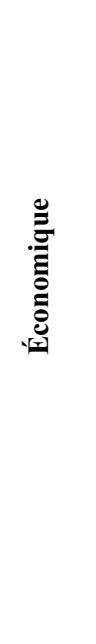 } & Réduction des dépenses & $\begin{array}{l}\text { Rénovation des toitures (gains d'isolation thermique, } \\
\text { remplacement des chaudières à gaz par des panneaux } \\
\text { photovoltaïques), stockage des encres de couleurs primaires, } \\
\text { centre de tri intégré (valorisation économique des déchets } \\
\text { papier), bambouseraie de phytoremédiation }\end{array}$ \\
\hline & Gains de productivité & $\begin{array}{l}\text { Machines d'emballage automatique des enveloppes, } \\
\text { technologie d'enroulage brevetée }\end{array}$ \\
\hline & $\begin{array}{l}\text { Amélioration du service au } \\
\text { client }\end{array}$ & $\begin{array}{l}\text { Technologie d'enroulage brevetée, enveloppes } \\
\text { transpromotionnelles (principe du } 2 \text { en } 1 \text { ) }\end{array}$ \\
\hline & $\begin{array}{l}\text { Réponse marketing à une } \\
\text { demande croissante }\end{array}$ & $\begin{array}{l}\text { Enveloppes éco-compatibles (produits écoresponsables), } \\
\text { pochettes personnalisables (personnalisation de l'offre) }\end{array}$ \\
\hline & Effet de réputation & $\begin{array}{l}\text { Association pour le reboisement, technologie d'enroulage } \\
\text { brevetée (image de pionnier), enveloppes } \\
\text { transpromotionnelles (pionnier) }\end{array}$ \\
\hline & Diversification & Activités de conseil en DD \\
\hline \multirow{6}{*}{ 吾 } & $\begin{array}{l}\text { Amélioration des conditions } \\
\text { de travail des opérateurs }\end{array}$ & $\begin{array}{l}\text { Machines d'emballage automatique des enveloppes, } \\
\text { technologie d'enroulage brevetée; stockage des encres } \\
\text { (réduction des risques), récupération des déchets papier } \\
\text { (réduction des poussières), rénovation des toitures (luminosité } \\
\text { naturelle), création d'espaces verts (bambouseraie) }\end{array}$ \\
\hline & $\begin{array}{l}\text { Développement } \\
\text { des compétences }\end{array}$ & $\begin{array}{l}\text { Machines d'emballage automatique des enveloppes (métiers } \\
\text { du contrôle qualité), technologie d'enroulage brevetée }\end{array}$ \\
\hline & $\begin{array}{l}\text { Implication des salariés dans } \\
\text { le projet d'entreprise }\end{array}$ & $\begin{array}{l}\text { Peinture des machines des ateliers aux couleurs des marques } \\
\text { de la PME, intéressement aux résultats de l'entreprise }\end{array}$ \\
\hline & $\begin{array}{l}\text { Diffusion des bonnes } \\
\text { pratiques de DD }\end{array}$ & Activités de conseil auprès des PME régionales \\
\hline & $\begin{array}{l}\text { Projet d'insertion profession- } \\
\text { nelle des prisonniers }\end{array}$ & Pochettes d'emballage personnalisables \\
\hline & Prise en compte des riverains & $\begin{array}{l}\text { Rénovation des toitures (réduction des nuisances sonores), } \\
\text { association pour le reboisement (implication des populations } \\
\text { locales dans les actions sur le terrain) }\end{array}$ \\
\hline
\end{tabular}


d'amélioration continue est légitime car elles impliquent la partie prenante la plus immédiate de l'entreprise, à savoir ses salariés (Hammann et al., 2009) et représentent des coûts estimés entre 20 et $30 \%$ de la masse salariale des entreprises. Par ailleurs, les horaires de début et de fin des équipes sont décalés pour éviter les embouteillages fréquents dans la métropole lilloise. Ceci réduit le stress des salariés et contribue in fine à une meilleure performance de l'organisation. En résumé, les trois volets du DD sont omniprésents dans les innovations tant d'exploration que d'exploitation de la PME (tableau 1).

La question de la dimension temporelle des relations entre innovations est centrale dans la littérature (Ayerbe, 2006), d'où une approche chronologique de la conciliation d'innovations d'exploration et d'exploitation dans la PME (figure 1). À l'instar des résultats d'Ayerbe (2006) sur les relations entre innovations technologiques et organisationnelles en milieu PME, on constate une co-activation entre innovations d'exploration et d'exploitation, mais aussi une accélération du rythme des innovations depuis l'obtention de la triple certification QSE en 2008. Celle-ci a pu jouer un rôle de catalyseur de la capacité dynamique d'innovation au sein de la PME par une volonté toujours plus affirmée de concilier les trois piliers du DD, comme le montre cet extrait de la politique QSE 2010-2011.

\section{POLITIQUE QSE 2010-2011 (EXTRAIT)}

Nous agissons sur trois plans principaux que nous déclinons pour chaque activité : La réduction de notre impact sur l'environnement et la prévention des pollutions. La réduction du risque au travail et la baisse de la pénibilité des postes. L'amélioration de la productivité de l'activité et du site industriel.

Figure 1 - Concilier innovations d'exploration et d'exploitation orientées DD dans une PME

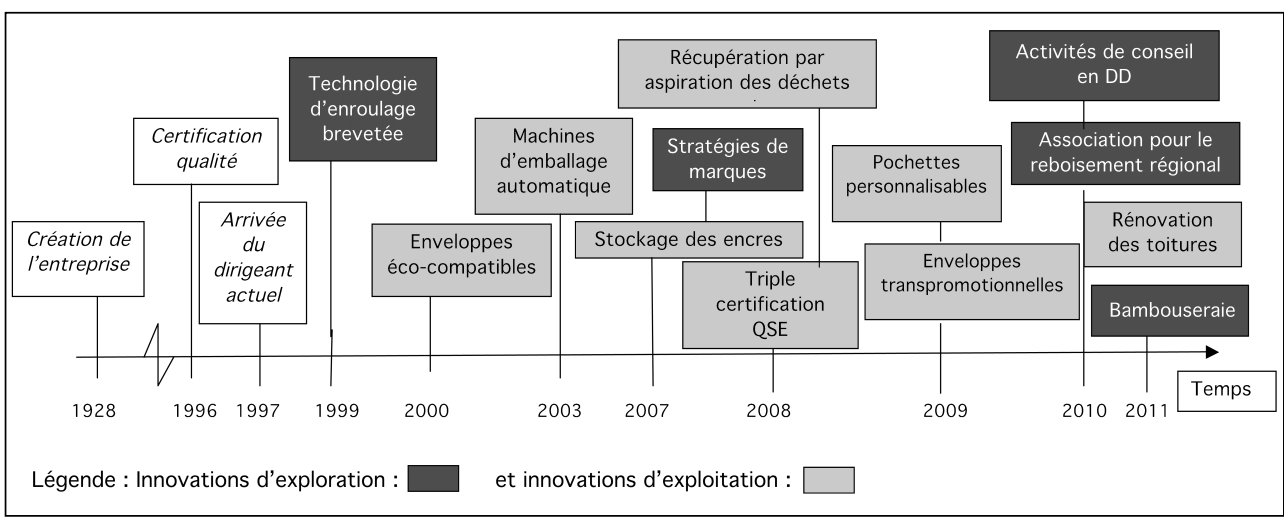




\section{Une posture entrepreneuriale au service de la stratégie d'innovation durable}

Le dirigeant de la PME peut légitimement être qualifié d'innovateur au sens de Miles et Snow (1978) dans la mesure où il a à cœur non seulement d'entreprendre autrement au quotidien (avec le DD comme fil directeur), mais également de piloter les changements induits dans la durée par la mise en œuvre de ses innovations. Depuis 1997, c'est un entrepreneur militant qui dirige cette PME : « Nous sommes guidés par des idées, des convictions, notre patron est un militant » (responsable QSE), signe du partage des valeurs du dirigeant, ce qui constitue un point névralgique du management responsable (Murillo et Lozano, 2006 ; Spence, 2007). Comme l'explique le dirigeant, véritable " champion interne » (Jenkins, 2006) : « Je n'ai pas de certitude. On se forge des convictions au fil des années. Pour moi, c'est insupportable d'avoir des travailleurs pauvres, surendettés. Je parle de «non-assistance à planète en danger », de «non-assistance à salarié en danger ». Moi, je mets ma révolte au service de mon engagement, c'est mon moteur pour avancer, microagir... » Dans une philosophie proche de l'entreprise apprenante (Senge, 1991), le dirigeant explique : « Je n'ai pas la prétention de tout réinventer, mais juste d'aller dans le décalage en regardant les choses sous un autre angle », signe d'une volonté d'apprentissage permanent en conformité avec le modèle de Jenkins (2009). L'ouverture vers l'extérieur de la PME est recherchée par l'entrepreneur, mais avec discernement, car l'implication formelle dans les réseaux professionnels n'est pas une priorité affichée, ce qui le distingue des propositions de Jenkins (2009). Le dirigeant de la PME s'apparente à un entrepreneur visionnaire, un véritable agent du changement tel que préconisé par Jenkins (2009) : « Nous ne faisons pas du DD parce que c'est à la mode, mais bien parce que c'est une préoccupation qui occupe mes décisions depuis mon investiture. » (dirigeant). Ceci renvoie aux travaux de Jenkins (2009) pour qui la RSE est de moins en moins un "plus », mais simplement «la manière de faire les choses" (p. 34). Le caractère profondément humaniste du dirigeant transpire dans la stratégie de son entreprise. Pour autant, il reste pragmatique : « Pour moi, l'argent n'est pas une fin en soi, mais un moyen d'entreprendre. Chez nous, les salaires vont de 1 à 4,5 . Les plus bas salaires sont $15 \%$ au-delà de la moyenne de la profession ; c'est normal, on est $15 \%$ plus productifs que la moyenne. Du coup, on est bien notés par la Banque de France, ce qui nous permet d'obtenir des financements bancaires à des taux plus intéressants. Tout ça, c'est un cercle vertueux. » (dirigeant). Cependant, le dirigeant admet que les bons résultats économiques de la PME ne découlent pas directement de son management responsable: " on n'a jamais remporté un contrat parce qu'on était plus écologique... l'économique prime $»^{17}$. La ligne directrice demeure sans équivoque le rapprochement entre écologie et économie sous condition de profitabilité pour l'entreprise.

La vision stratégique du dirigeant guide ses actions et s'émancipe du déterminisme environnemental : «Idéalistes, nous le

17. Témoignage du dirigeant lors du salon Jadde 2010 à Lille. 
sommes et je le revendique. L'idéal est un guide, un fil rouge qui oriente notre stratégie sur le long terme. Regarder un idéal, c'est se fixer un objectif, lointain et élevé » (dirigeant). Sa personnalité permet un management résolument participatif : les échanges informels sont privilégiés et les regards neufs systématiquement suscités par l'ouverture d'esprit du dirigeant. Cette quête de créativité renvoie à l'un des leviers du modèle de Jenkins (2009) pour identifier des opportunités durables. En résumé, la posture entrepreneuriale du dirigeant de la PME s'inscrit dans un régime d'innovation continue, régime soutenu par des valeurs humanistes prônées par Jenkins (2009) comme socles d'un nouveau modèle économique basé sur la RSE.

\section{CONCLUSION}

Cet article illustre la manière dont le DD peut constituer un levier d'innovation dans une PME en dépassant une approche purement palliative de gestion des risques (industriels, environnementaux, de santé au travail) au profit d'une proactivité affirmée. Le choix d'une analyse par la capacité dynamique d'innovation (Chanal et Mothe, 2005) contribue à l'enrichissement des connaissances en matière de management responsable en milieu PME : «C'est par l'innovation et la qualité de nos produits que nous restons compétitifs, mais nos clients recherchent avant tout le moins cher. Nous essayons de garder en tête ces deux critères : être compétitif et rester vertueux dans notre façon de travailler ». Autrement dit, la stratégie de la PME s'inscrit dans la création de valeur partagée au sens de Porter et Kramer (2011), notion constituant le fil directeur de notre analyse. Dans cette optique, les capacités dynamiques jouent bien un rôle clé dans la poursuite d'un avantage concurrentiel (Easterby-Smith et al., 2009). L'analyse de la capacité dynamique d'innovation de cette PME conforte également l'idée selon laquelle la mise en œuvre d'une stratégie environnementale proactive favorise l'émergence d'innovations de natures différentes (Bianchi et Noci, 1998). La limite de ce travail est inhérente à sa méthodologie basée sur un cas unique de PME, sans visée de généralisation. Notre ambition était modestement de montrer l'intérêt d'une approche en termes de capacité dynamique d'innovation durable dans un contexte de PME. La PME étudiée est à bien des égards " exemplaire » au sens de Jenkins (2006), ce qui n'exclut pas le risque de rationalisations a posteriori induites par les analyses de discours des acteurs. Néanmoins, son dirigeant n'est ni un philanthrope ni un utopiste, mais simplement un individu soucieux de contribuer à son échelle au mieux-être dans la société tout en pérennisant son activité. Ainsi, la diversification vers les activités de conseil en DD relève autant d'un engagement sincère de diffusion de bonnes pratiques que de la nécessité économique d'anticiper les mutations du marché de l'enveloppe.

Sur le plan managérial, les entrepreneurs ont intérêt à prendre conscience du virage vers une économie verte dans la lignée des travaux de Chanal (2002) sur l'accompagnement des PME dans leur processus d'innovation. Cela implique une sensibilisation accrue des organismes d'accompagnement des dirigeants de PME à ces questions de $\mathrm{DD}$ en les incitant à les intégrer davantage dans les plans d'affaires des porteurs de projet (initiatives du Réseau Entreprendre ${ }^{\circledR}$ depuis fin 2008). À titre d'exemple, le pro- 
gramme transfrontalier franco-belge $\mathrm{IODDE}^{18}$ vise à sensibiliser et accompagner les PME dans l'identification d'opportunités d'affaires basées sur l'innovation et le DD. En raison de son exemplarité, la PME analysée dans notre article a participé au séminaire introductif de ce programme en mars 2011, signe de son caractère reconnu de « championne de RSE » (Jenkins, 2006). Son dirigeant a ainsi valorisé le savoir-faire de la PME en matière de management responsable par la présentation chiffrée des économies réalisées. Parmi les pistes de recherche futures, deux retiennent notre attention: 1) l'analyse du processus de co-activation (Ayerbe, 2006) des innovations d'exploration et d'exploitation et 2) l'étude des parties prenantes des PME comme des réseaux à signaux faibles porteurs d'innovation (Labelle, 2008).

\section{BibliograPHIE}

Abrassart C., Aggeri F., "Quelles capacités dynamiques pour les stratégies de développement durable des entreprises ? Le cas du management de l'éco-conception ", Actes de la XVI Conférence de l'AIMS, Montréal, 2007.

Aragon-Sanchez A., Sanchez-Marin G., "Strategic orientation, management characteristics and performance: A study of Spanish SMEs", Journal of Small Business Management, vol. $43, n^{\circ} 3,2005$, p. 287-308.

Ayerbe C., «Innovations technologique et organisationnelle au sein de PME innovantes : complémentarité des processus, analyse comparative des mécanismes de diffusion », Revue Internationale PME, vol. 19, $\mathrm{n}^{\circ}$ 1, 2006, p. 9-34.

Barreyre P.Y., La stratégie d'innovation dans les moyennes et petites industries, Éditions Hommes et Techniques, Paris, 1975.

Bellini B., «Un nouvel enjeu pour l'entreprise: la prise en compte de la protection de l'environnement dans son management. État des lieux et perspectives», Actes $d u$ Premier Atelier DD de l'AIMS, Angers, 15 mai, 2003.

Bianchi R., Noci G., "Greening SMEs competitiveness", Small Business Economics, n 11, 1998, p. 269-281.

Camison-Zomosa C., Lapiedra-Alcani R., Segarra-Ciprés M. et Boronat-Navarro M., "A meta-analysis of innovation and organizational size", Organization Studies, vol. 25, n 3, 2004, p. 331-361.

Castiaux A., « Responsabilité d'entreprise et innovation : entre exploration et exploitation », Reflets et Perspectives, vol. XLVIII, $n^{\circ}$ 4, 2009, p. 37-49.

Chanal V., "Comment accompagner les PME-PMI dans leur processus d'innovation ? », Actes de la XI Conférence de l'AIMS, Paris, 2002.

Chanal V., Mothe C., "Concilier innovations d'exploitation et d'exploration. Le cas du secteur automobile », Revue française de gestion, $n^{\circ} 154,2005$, p. 173-191.

18. IODDE : Innovations et opportunités de développement durable pour votre entreprise. (www.iodde.net). Ce programme, soutenu par l'Union européenne dans le cadre d'INTERREG, propose un accompagnement personnalisé suite à un diagnostic et à des formations collectives sur diverses thématiques comme l'éco-conception. 
Conceiçao P., Heitor M.V. et Vieira P.S., "Are Environmental Concerns Drivers of Innovation? Interpreting Portuguese Innovation Data to Foster Environmental Foresight", Technological Forecasting and Social Change, vol. 73, n 3, 2006, p. 266-276.

Damanpour F., "Organizational Innovation: A meta-analysis of Effects of Determinants and Moderators”, Academy of Management Journal, vol. 34, n 3, 1991, p. 555-590.

Duncan R.B., "The Ambidextrous Organization: designing Dual Structures for Innovation, The Management of Organization", Killman R.H., Pondy L.R., Slevin D., North Holland, New York, 1976.

Easterby-Smith M.P.V., Lyles M., Peteraf M., "Dynamic Capabilities: Current Debates and Future Directions", British Journal of Management, vol. 20, 2009, p. 51-58.

Eisenhardt K.M., Martin J.A., "Dynamic Capabilities: What are They?", Strategic Management Journal, vol. 21, 2000, p. 1105-1121.

Gupta A.K., Smith K.G., Shalley A.C., "The Interplay between Exploration and Exploitation", Academy of Management Journal, vol. 49, n 4, 2006, p.693-706.

Grayson D., Hodges A., Corporate Social Opportunity! Seven Steps to Make Corporate Social Responsibility Work for Your Business, Greenleaf Publishing, London, 2004.

Hammann E.M., Habisch A., Pechlaner H., "Values that create value: socially responsible business practices in SMEs - empirical evidence from German companies", Business Ethics: A European Review, vol. 18, $\mathrm{n}^{\circ}$ 1, 2009, p. 37-51.

Hart S., "A natural resource based view of the firm", Academy of Management Review, vol. 20, 1995, p. 986-1014.

Hlady-Rispal M., La méthode des cas - Application à la recherche en gestion, De Boeck Université, Bruxelles, 2002.

Jenkins H., "A critique of conventional CSR theory: an SME perspective", Journal of General Management, vol. 9, $\mathrm{n}^{\circ}$ 4, 2004, p. 55-75.

Jenkins H., "A business opportunity model of corporate social responsibility for small-and medium-sized enterprises", Business Ethics: A European Review, vol. 18, n 1, 2009, p. 21-36.

Julien P.A., Les PME : bilan et perspectives, Economica, Paris, 1997.

Julien P.A., Lachance R., « Colombo, Holmes, Maigret, de Baskerville et l'entrepreneuriat régional », La stratégie dans tous ses états, Fourcade C., Paché G., Perez R., chap. 22, Éditions EMS, Caen, 2006.

Kramer M., Pfizer M., Lee P., "Competitive Social Responsibility: Uncovering the Economic Rationale for Corporate Social Responsibility among Danish Small and Medium-Sized Enterprises", Project Report, Copenhague, 2007.

Labelle F., «La PME et la durabilité : une gestion qui améliore les capacités d'innovation des entrepreneurs », Actes du IX CIFEPME, Louvain la Neuve, Belgique, 2008.

Le Bas C. et Poussing N., " Existe-t-il une relation entre RSE/innovation ? Exploitation empirique sur données luxembourgeoises », Working Paper CEPS Instead, $n^{\circ}$ 2010-11, 2010. Little A.D., "The Innovation Highground - Winning tomorrow's Customers Using Sustainability driven Innovation", Strategic Direction, n 22, 2006, p. 35-37. 
McWilliams A., Siegel D.S., Wright P.M., "Corporate social responsibility: strategic implications", Journal of Management Studies, vol. 43, n 1, 2006, p.1-18.

MacLagan P., "Corporate social responsibility as a participative process", Business Ethics: A European Review, vol. 8, n 1, 1999, p. 43-49.

March J., "Exploration and Exploitation in Organizational Learning", Organization Science, vol. 2, $\mathrm{n}^{\circ} 1,1991$, p. 71-87.

Martinet A.C., Payaud M., «Formes de RSE et entreprises sociales. Une hybridation des stratégies », Revue française de gestion, $\mathrm{n}^{\circ}$ 180, 2008, p. 199-214.

Martinet A.C., Reynaud E., Stratégies d'entreprise et écologie, Economica, Paris, 2004.

Mathieu A., "Écostratégies et réponse industrielle aux attentes sociétales: quelles implications ?», Actes du $3^{e}$ Atelier DD de l'AIMS, Lyon, 2008.

Mathieu A., Soparnot R., «L'appropriation du concept de développement durable en entreprise : un générateur d'innovation », Actes de la XVI Conférence de l'AIMS, Montréal, 2007.

Mendibil K., Hernandez J., Espinach X., Garriga E., McGregor S., "How Can CSR Practices Lead to Successful Innovation in SMEs?", Publication from the RESPONSE Project, 2007, cités par A. Castiaux, 2009.

Miles R., Snow C.C., Organizational Strategy, Structure and Process, Mc Graw Hill, New York, 1978.

Moore G., Spence L., "Editorial: Responsibility and Small Business", Journal of Business Ethics, n 67, 2006, p. 219-226.

Morsing M., Perrini F., "CSR in SMEs: do SMEs matter for the CSR agenda?", Business Ethics: A European Review, vol. 18, n 1, 2009, p. 1-6.

Murillo D., Lozano J.M., "SMEs and CSR: An Approach to CSR in their Own Words", Journal of Business Ethics, n 67, 2006, p. 227-240.

Nielsen A.E., Thomsen C., "Investigating CSR communication in SMEs: a case study among Danish middle managers", Business Ethics: A European Review, vol. 18, n 1, 2009, p. 83-93.

Nidumolu R., Prahalad C.K., Rangaswami M.R., "Why Sustainability is now the Key Driver of Innovation?”, Harvard Business Review, vol. 87, 2009, p. 57-64.

Observatoire des PME européennes, Les PME européennes et les responsabilités sociale et environnementale, Rapport $\mathrm{n}^{\circ}$ 4, 2002.

Patris C., Valenduc G., Warrant F., « L'innovation technologique au service du développement durable », Synthèse $d u$ rapport final du programme "Leviers $d u$ développement durable », Bruxelles, 2001.

Porter M.E., “America’s green strategy”, Scientific American, vol. 264, 1991, p. 168.

Porter M.E., Van der Linde C., "Towards a new conception of the environment: competitiveness relationship", Journal of Economic Perspectives, vol. 9, n 4, 1995, p. 97-118.

Porter M.E., Kramer M.R., "Strategy and Society”, Harvard Business Review, 2006, p. 77-92.

Porter M.E., Kramer M.R., "Creating Shared Value”, Harvard Business Review, JanuaryFebruary 2011, p. 62-77. 
Rennings K., Ziegler A., Ankele K., Hoffmann E., "The Influence of Different Characteristics of the EU Environmental Management and Auditing Scheme on Technical Environmental Innovations and Economic Performance", Ecological Economics, ${ }^{\circ} 57$, 2006, p. 45-59.

Schumpeter J., Théorie de l'évolution économique, Dalloz, Paris, 1935.

Senge P., La cinquième discipline, Editions First, Paris, 1991.

Soparnot R., Stevens E., Management de l'innovation, Dunod, Paris, 2007.

Spence L.J., "CSR and Small Business in a European Policy Context: The Five 'C' of CSR and Small Business Research Agenda 2007”, Business and Society Review, vol. 112, n 4, 2007, p. 533-552.

Teece J., Pisano G., Shuen A., "Dynamic Capabilities and Strategic Management”, Strategic Management Journal, vol. 18, $\mathrm{n}^{\circ}$ 7, 1997, p. 509-533.

Wacheux F., Méthodes qualitatives et recherche en gestion, Economica, Paris, 1996.

Yin R.K., Case Study Research. Design and Methods, Sage Publications, London, 1994. 